\title{
Manufacturing MSCs for commercial application: an interview with Ross Macdonald
}

\author{
Ross Macdonald*,1 \\ ${ }^{1}$ Cynata Therapeutics, 62 Lygon Street, Carlton, VIC 3053, Australia \\ *Author for correspondence: ross.macdonald@cynata.com
}

Ross Macdonald is the CEO and Managing Director of Cynata Therapeutics Limited (Australia). He has over 30 years of experience and a track record of success in pharmaceuticals and biotechnology businesses. His career history includes positions as Vice President of Business Development for Sinclair Pharmaceuticals Ltd (now Sinclair IS Pharma), a UK-based specialty pharmaceuticals company, and Vice President of Corporate Development for Stiefel Laboratories, Inc., then the largest independent dermatology company in the world and acquired by GlaxoSmithKline in 2009 for $£ 2.25$ billion. He has also served as the CEO of Living Cell Technologies Ltd, Vice President of Business Development of Connetics Corporation and Vice President of Research and Development of F H Faulding \& Co Ltd.

First draft submitted: 13 June 2019; Published online: 7 November 2019

The interview was conducted by Adam Price-Evans, Managing Commissioning Editor of Regenerative Medicine.

Cynata's technology, Cymerus, is a unique approach to the manufacture of therapeutic mesenchymal stem/stromal cells from induced pluripotent stem cells. Could you tell me a little more about this?

Historically, the approach to manufacturing therapeutic mesenchymal stem/stromal cells (MSCs) has involved extracting a tissue source containing a population of MSCs and employing purification technology to obtain a purified MSC population. Unfortunately, due to the heterogeneous nature of these tissue sources, MSCs are very scarce. Given that a single dose of MSCs is typically around one million cells per kilogram, an average dose in an adult male would require around 80-100 million cells. A bone marrow aspirate, however, only yields approximately 20,000 authentic MSCs, which means that you have to expand the cells in culture to obtain the desired number. We now know, however, that MSCs undergo replicative senescence; in other words, they have a limited capacity for cell replication and renewal. When this capacity is exceeded, they start to divide less frequently and lose their potency, so when it comes to industrializing the manufacturing of these products for commercial application, there is a serious manufacturing challenge.

Given the controversy surrounding embryonic stem cells making commercial application very difficult, we looked toward induced pluripotent stem cells (iPSCs). These cells provide a non-controversial option for obtaining a starting material capable of infinite cell renewal, offering the consistency necessary for a pharmaceutical product. The iPSC approach therefore gives us a tool that addresses the manufacturing challenge associated with tissuederived MSCs. Our technology involves accessing an iPSC line that we can manufacture infinitely and then differentiate those iPSCs into MSCs, thus ensuring that those MSCs are fresh, robust and all are exact copies of one another. This means that, in theory, whatever results you get in a clinical trial today will be the same results you get next month and next year.

\section{How do the iPSC-derived MSCs compare to standard MSCs? Do they behave mechanistically in the same manner?}

It is actually a very interesting situation at the moment in the field due to the big divide about what exactly constitutes an MSC. The International Society for Cellular Therapy has provided a rather broad set of defining characteristics and within the boundaries of that loose definition, our MSCs share all of those properties, technically

Future : Medicine 
making them 'genuine' MSCs. In fact, I believe they resemble freshly isolated MSCs because they are much more potent than MSCs that have suffered replicative senescence following repeat culture.

There is, however, an ongoing debate about exactly what an MSC is. My position is that once you have established that you can make a consistent product and therapeutic outcome showing safety and efficacy, it does not really matter what you call them. The fact that aspirin is called aspirin is only a quirk of nomenclature. The interesting issue is that while aspirin is confined by a chemical structure making it easy to define, a living cell's characteristics could be quite different from one minute to the next. I do not think this will ever get fully resolved as there will probably always be a debate in a biological setting due to interest in how MSCs develop in the body. As products move into a commercial setting, I believe the debate will become less relevant, since doctors prescribing the product probably will not care whether it is an MSC; they'll only care that it is a safe and effective treatment for the disease in question.

\section{Are there any difficulties with the Cymerus technology \& the use of iPSC-derived MSCs?}

While I may be biased, I do not think there are any particular difficulties with this technology. The original process that was used to derive iPSCs involved genomic integration of the reprogramming factors, which was not ideal for therapeutic purposes as it raises questions over safety. The procedure in use now no longer involves changing the genetic structure of the target cell, so a lot of those safety concerns are alleviated. Of course, there is still the challenge of ensuring the finished product contains no starting material, in other words, pluripotent cells, but our manufacturing processes are very stringent from both a quality assurance and quality control perspective, which has enabled us to satisfy the regulatory agencies. The general complexity of creating iPSC lines may have also been a stumbling block in the past; however, now that we have our cell line, this has also become a moot point for us. There are also those that are against the use of iPSCs in general, but as time passes and the biology of iPSCs becomes clearer and study results showing safety and efficacy are released, I think this negative voice will become diminished.

\section{Can you tell me about the preclinical work you carried out with this technology in graft-versus-host disease before moving toward clinical trial?}

We were fortunate in that much of the early therapeutic work and many of the groundbreaking clinical trials with MSCs were actually carried out in graft-versus-host disease (GvHD) patients. We therefore had a well-worn path that we could follow in conducting our own clinical study. We also had a good idea on what would be required from a preclinical safety package for approval from regulatory agencies to proceed. In essence, we started with a standard set of preclinical studies that confirmed the potency of our cells and the consistency of the product. We then conducted a preclinical study on a GvHD animal model to demonstrate that this product was effective in treating animals with GvHD. All of that data formed the basis of our application to the regulatory agency, in this case the Medicines and Healthcare Regulatory Agency in the UK, to commence a clinical study, which was accepted, allowing us to move forward into our clinical program in 2017.

\section{Why did you choose to focus on GvHD specifically?}

The aforementioned breadth of research already carried out in the area was certainly one of the reasons, as it meant we did not have to reinvent the wheel. We already knew that robust and potent MSCs were very effective in treating GvHD patients, so that burden of proof was perhaps a little less challenging than it normally would be. Another important factor in our decision was that these patients had no other recourse, as they had failed the standard of care (i.e., corticosteroid treatment). Most patients in this position unfortunately suffer death within 1-2 years of diagnosis and so from a regulatory point of view, it is less challenging than if your product is for treating a 'non-life-threatening' disease like ingrown toenails. The regulators will consider the risk-benefit in that context. A third important element was the duration of the study and the end points. Being a small Australian company with limited access to capital, we did not want to conduct a study that would take a long time with vague end points. We essentially needed a quick 'shot on goal' and a quick result so that we could confirm (or otherwise) whether our product was safe and effective.

One negative element that we had to consider when choosing to focus on GvHD was the commercial attractiveness. Thankfully, GvHD is quite a rare disease, however, this means that commercially speaking, it is not terribly appealing. We knew from the beginning that we are not going to make a lot of money from focusing on GvHD, but we were not too concerned because our goal was to ensure we had a product that worked. 


\section{You have now completed the first Phase I clinical trial using allogenic iPSC-derived MSCs. Can you tell me about the trial design \& the key results obtained from this study?}

GvHD patients that had failed the standard of care were recruited via open recruitment both in the UK and Australia. In total, we treated 15 adults in two cohorts: a low dose group initially and a high dose group subsequently. Patients received two doses of cells, seven days apart, and then we assessed the end points at 28 and 100 days following the first dose. The primary end points were safety and tolerability, as with any Phase I clinical trial, and the secondary end points were essentially efficacy end points of complete response, partial response and overall survival.

Unlike many Phase I trials that typically involve healthy volunteers, we conducted this study in patients with the target disease. When you conduct a typical Phase I trial in healthy volunteers, you are looking to show that the treatment is safe, but you do not really know if the treatment might work until you conduct a Phase II trial. This means, from an investment point of view, the valuation will not have changed a great deal. However, in studies such as ours that involve treating actual patients, you do get a better indication of whether the therapy may be commercially successful in the future. This was obviously an important point from an investor's point of view, but also for the patients themselves.

The study met all of the primary end points and secondary end points, showing a clean safety and tolerability profile and a complete response (i.e., complete resolution of symptoms) in just over half of the patients. Given that these patients normally just continue to go downhill and do not ever get better, this was an outstanding result. We saw an overall response rate (i.e., an improvement by at least one GvHD grade) in just under $90 \%$ of the patients. We believe the only reason we did not see it in all patients was that two patients were not available for a follow-up; one patient died of pneumonia, an unfortunate consequence of GvHD, and one patient withdrew from the study for personal reasons. There was also an over $87 \%$ overall survival rate. Together, we felt these were truly outstanding results, especially when you consider that they were obtained following only two doses of the product, compared with other tested GvHD products that required at least eight doses. This is definitely beneficial because not only is it more convenient for the patient, but it is also advantageous from a cost-of-goods perspective.

\section{Could you tell me about any plans for a Phase II trial in GvHD?}

We recently announced that our partner, Fujifilm Corporation, has exercised a license option and now has exclusive, worldwide rights to develop, manufacture and market CYP-001, Cynata’s lead Cymerus MSC product, for the treatment of GvHD. Fujifilm plans to start a company-sponsored phase II clinical trial in Japan before the end of 2020. Cynata will potentially receive additional future milestone payments from Fujifilm totaling up to US $\$ 43 \mathrm{~m}$ based on successful attainment of certain industry standard product development and commercial milestones, the first of which is on completion of the first phase II clinical trial. Subsequent milestones are the completion of phase III clinical trials, submission of applications for regulatory approvals, acceptance of geographic marketing authorizations, first sales and extending the indication.

\section{You are also studying this technology for use in other indications such as osteoarthritis \& critical limb ischemia. Could you tell me about this work \& the next steps?}

Both autologous and off-the-shelf allogenic MSC products have been investigated in active trials around the world for these indications so, as with GvHD, we will be following a well-trodden path.

Critical limb ischemia (CLI) represents a very large medical need and we have some compelling preclinical data using a CLI model. The planning for a Phase II trial, which will be conducted in the UK and Australia, is currently underway, which will investigate end-stage CLI patients whose most likely outcome is an amputation in the next 12-24 months.

For osteoarthritis, we were actually approached by an academic group in Australia who have long been looking at the potential utility of MSCs in osteoarthritis but have encountered many challenges due to the variability of the MSC products that they were using. Using our cells, they plan to complete a 450-patient Phase II clinical study to definitively answer whether MSCs are effective in treating this condition. This will be one of the largest trials ever undertaken with MSCs and will be largely funded by the Australian Government. 
Finally, how do you think the field of regenerative medicine will look in 15 years? What do you think are the key challenges that the field will have to overcome before these therapies reach the clinic?

First, there is the manufacturing challenge which remains ultimately unmet. It should be noted however that when monoclonal antibodies, for example, were first identified and proposed as a 'magic bullet' therapeutic, most of the industry said, "Are you mad? They are very hard to make and are incredibly expensive. How would you ever get a pharmaceutical product out of an academic curiosity?" Ultimately, the manufacturing solutions were proposed and now, the rest is history. 25 years ago, no one would have ever considered that possible. Similar concerns are being raised today with cell therapy and regenerative medicine products. Generally, they are still very hard to make, they are complex, there are reproducibility issues and they are incredibly expensive. Ultimately, as with monoclonal antibodies, there will be manufacturing approaches, perhaps similar to ours, that will lead to a more viable commercial pathway for these products in the future.

The second point is data, data, data. Early positive results in the MSC field offered great promise, but this was met with disappointment when these results were not replicated in Phase II or III trials. This led to many challenges in our sector, largely related to the inability to gain sufficient investment capital. I think that this will change once we start to see more compelling data. Aside from our own trial in GvHD, there are other products around the world moving forward and as more convincing evidence is presented, I am sure we will see that typical wave of excitement start to crest again and there will be sustainable products going through to market. The failures, unfortunately, are a real setback but that is just part and parcel of drug development.

Financial \& competing interests disclosure

R Macdonald is a paid employee (Chief Executive Officer) of Cynata Therapeutics Ltd and is a shareholder in that company. The author has no other relevant affiliations or financial involvement with any organization or entity with a financial interest in or financial conflict with the subject matter or materials discussed in the manuscript apart from those disclosed.

No writing assistance was utilized in the production of this manuscript.

Disclaimer

The opinions expressed in this interview are those of R Macdonald and do not necessarily reflect the views of Future Medicine Ltd. 